US Army Corps of Engineers

\section{Evaluation of Colorimetric Methods for Measuring Reduced (Ferrous) Iron}

by Stephen P. Faulkner, Paul E. Hintze, and Steven L. Ashby

PURPOSE: This technical note describes a comparative study of colorimetric methods for real-time measurements of ferrous iron in reservoir tailwaters. Two methods were evaluated: the 1,10 phenanthroline method for the Hach DR/2000 spectrophotometer (Hach Company, Loveland, $\mathrm{CO}$ ) and a laboratory method developed for field application using 3-(2-pyridyl)-5, 6-bis(4-phenylsulfonic acid)-1,2,4-triazine (FerroZine) ${ }^{1}$ and a portable Milton Roy Mini 20 spectrophotometer.

BACKGROUND: Reduced, or ferrous, iron forms in aquatic systems as dissolved oxygen levels (and therefore the oxidation reduction potential) decrease to levels sufficient to allow reduction from oxidized (ferric) iron. This is a common occurrence in the deeper regions of reservoirs and lakes. Ferrous iron is soluble, and concentrations in water overlying the sediments where reduction has occurred increase under hypoxic conditions (low concentrations of dissolved oxygen). Diffusion of the soluble ferrous iron throughout the deeper waters results in elevated concentrations in the release and downstream area (tailwater). As release waters are aerated via outlet works, stilling basins, and aeration in the tailwater, the reduced iron is quickly oxidized to ferric iron and forms particulates that are subject to suspension, settling, and resuspension in the tailwater depending upon velocity and characteristics of the particles.

The rate of oxidation is quite rapid, and real-time measurements of oxidized and reduced concentrations are necessary if assessments of kinetics for modeling, particulate formation, or concentration changes are to be conducted. Oxidized iron is usually calculated as the difference between total and reduced iron since these can be measured specifically. Total iron can be determined with a variety of methods (atomic adsorption spectroscopy, inductively coupled plasma, and even colorimetrically), usually following a rigorous acid digestion. Several colorimetric methods are available for measurement of reduced iron but vary somewhat in their applicability (for example, ease of use, concentration range, recovery). Filtration (with filters of various pore sizes) has also been used to separate particulate iron from soluble iron for quantifying oxidized and reduced iron, respectively. However, though this may not be an appropriate indication of oxidation state (Dortch, Tillman, and Bunch 1992).

Previous studies (Faulkner, Gambrell, and Ashby 1996) indicated that colorimetric methods were better indicators of oxidized and reduced iron than using separation with filtration based on solubility. Among the colorimetric methods used, FerroZine was considered more appropriate at concentrations common to reservoir releases, although a' a',-dipyridyl was acceptable for analyses

1 Registered trademark of Hach Company, Loveland, CO. 
conducted within $26 \mathrm{hr}$. Stookey (1970) and Gibbs (1976) indicate that FerroZine can be used for specific determination of ferrous iron since the reagent forms a colored complex with ferrous but not ferric iron. The colorimetric method using FerroZine described by Hach Company (1992) employs a digestion prior to analysis which is not conducive to onsite analysis and is not described as specific for ferrous iron. Therefore, the Hach method using 1,10 phenanthroline, which is specific for determination of ferrous iron and does not require a digestion, was selected for comparison to commercially available 3-(2-pyridyl)-5, 6-bis(4-phenylsulfonic acid)-1,2,4-triazine (FerroZine).

The objective of this study was to evaluate the Hach method using 1,10 phenanthroline and a laboratory method using FerroZine with a portable spectrophotometer for the real-time analysis of reduced (ferrous) iron.

METHODS: Two colorimetric methods were used to measure ferrous iron concentrations in simulated reservoir water and water from a natural system. Tests were conducted at two concentration ranges (low, $2.5 \mathrm{mg} \mathrm{L}^{-1}$ as ferrous iron; high, $\sim 7.5 \mathrm{mg} \mathrm{L}^{-1}$ ) and at three conditions of dissolved oxygen concentrations (oxic, partially oxic, and anoxic). Each method was also tested on filtered $(0.4 \mu \mathrm{m})$ and unfiltered samples. Each sample was run in triplicate. Analysis for total iron was conducted using a Jarrell-Ash Atom Comp Series 800 Inductively Coupled Argon Plasma (ICP) spectrophotometer following filtration through a Whatman No. 42 filter and acidification with a drop of concentrated nitric acid.

Method 1 employed individual, premeasured reagent pillows containing 1,10 phenanthroline for color development and absorbance measurement on a Hach DR/2000 spectrophotometer. This method is easily applied in the field, does not require a standard curve, does not require preparation of any reagents, is specific for ferrous iron, and has a linear range of 0 to $3 \mathrm{mg} \mathrm{L}^{-1}$. Analyses were conducted per the manufacturer's guidelines (Hach Company 1992). Cuvettes were rinsed with deionized water $(0.1 \mathrm{~N} \mathrm{HCl})$, and a final time with deionized water between readings. Samples above the linear range were quantitatively diluted and reanalyzed. Method 1 was evaluated several times using the accuracy test provided in Hach Company (1992). The procedure requires preparation and analysis of a standard solution $\left(1 \mathrm{mg} \mathrm{L}^{-1}\right.$ concentration) using ferrous ammonium sulfate hexahydrate. Duplicate analyses were conducted immediately after preparation. A standard curve ranging from 0.4 to $3.0 \mathrm{mg} \mathrm{L}^{-1}$ ferrous iron was also analyzed in duplicate.

Method 2 employed the use of FerroZine in $50 \mathrm{mM}$ Hepes free acid to make a FerroZine reagent. The samples were analyzed by adding $1 \mathrm{ml}$ of reagent to $5 \mathrm{ml}$ of sample and then adding $4 \mathrm{ml}$ of deionized water. A standard curve was developed using a blank and standards containing 1, 2, 3, and $4 \mathrm{mg} \mathrm{L}^{-1}$ ferrous iron. Samples with concentrations greater than $4 \mathrm{mg} \mathrm{L}^{-1}$ were reanalyzed after dilution using $2 \mathrm{ml}$ of sample and $3 \mathrm{ml}$ of deionized water prior to addition of the reagent and additional deionized water.

The ability of each method to differentiate ferrous and ferric iron was evaluated using a solution of $2 \mathrm{mg} \mathrm{L}^{-1}$ ferrous iron (from ferrous ammonium sulfate) and $2 \mathrm{mg} \mathrm{L}^{-1}$ ferric iron (from ferric chloride). Analyses were conducted in triplicate immediately after preparation of the solution. The sample was then treated by bubbling air through the sample for $2.5 \mathrm{hr}$, and the $\mathrm{pH}$ was raised from 6 to 7.5 standard units to ensure that all iron was converted to the ferric form. The sample was then analyzed again in triplicate using both methods. 
Samples for ferrous determinations at both low and high concentrations were developed from simulated reservoir water and natural water (collected from the Amite River, Baton Rouge, LA). The natural waters were spiked with iron to increase concentrations to approximately 3.5 and $7.5 \mathrm{mg} \mathrm{L}^{-1}$, respectively. Concentrations of selected constituents for the low and high concentrations for the simulated reservoir water are presented in Table 1. The $\mathrm{pH}$ for both water sources was between 6.0 and 7.0 during the study, and any adjustments were made with $0.1 \mathrm{~N} \mathrm{HCl}$ or $\mathrm{NaOH}$ as necessary.

\begin{tabular}{|c|c|c|}
\hline Constituent & Low Concentration $\left(\mathrm{mg} \mathrm{L}^{-1}\right)$ & High Concentration $\left(\mathrm{mg} \mathrm{L}^{-1}\right)$ \\
\hline Iron & 2.5 & 7.5 \\
\hline Manganese & 2.5 & 7.5 \\
\hline Chloride & 3.5 & 10.5 \\
\hline Sulfate & 4.3 & 12.9 \\
\hline Fulvic acid & 2.5 & 2.5 \\
\hline
\end{tabular}

Different dissolved oxygen conditions were developed by bubbling air or nitrogen through the samples. Oxic conditions (dissolved oxygen concentrations between 11 and $12 \mathrm{mg} \mathrm{L}^{-1}$ ) were created by bubbling the air through the sample for at least $2 \mathrm{hr}$. Partially oxic samples were developed by bubbling nitrogen through the sample for $1 \mathrm{hr}$, then bubbling air through the sample for $10 \mathrm{~min}$. This treatment resulted in a dissolved oxygen concentration between 2.5 and $3.0 \mathrm{mg} \mathrm{L}^{-1}$. Anoxic samples were prepared by bubbling nitrogen through the samples for at least $2 \mathrm{hr}$, resulting in dissolved oxygen concentrations between 0.9 and $1.6 \mathrm{mg} \mathrm{L}^{-1}$.

\section{RESULTS AND DISCUSSION:}

- Accuracy of 1,10 Phenanthroline (Method 1). The standard curve used to evaluate the accuracy of 1,10 phenanthroline method (Method 1) indicates some loss in recovery (less than 10 percent at $2.0 \mathrm{mg} \mathrm{L}^{-1}$ and below) as concentrations increase (Figure 1), but accuracy was considered to be acceptable for measurements at $1.0 \mathrm{mg} \mathrm{L}^{-1}$ and below.

- Distinguishing Ferrous and Ferric Iron. Results of each method used to measure ferrous and ferric iron are presented in Figure 2. Both methods were considered to be specific for ferrous iron at time zero when ferrous and ferric were both present. At a period $2.5 \mathrm{hr}$ later, when oxidation of ferrous to ferric iron had occurred, ferrous iron measurements were $0.01 \mathrm{mg} \mathrm{L}^{-1}$ or less. As a result of these preliminary studies, both methods were considered to be capable of accurately measuring ferrous iron concentrations within \pm 10 percent at concentrations less than $3.0 \mathrm{mg} \mathrm{L}^{-1}$. The loss of measurable ferrous concentrations within $2.5 \mathrm{hr}$ suggests a need for rapid in situ analysis.

- Source Water, Concentration Effects, and Oxygen Conditions. Figures 3-8 present the results of concentration effects (low, in figure panels labeled A; high, in panels 
Water Quality Technical Note PD-02

March 1999

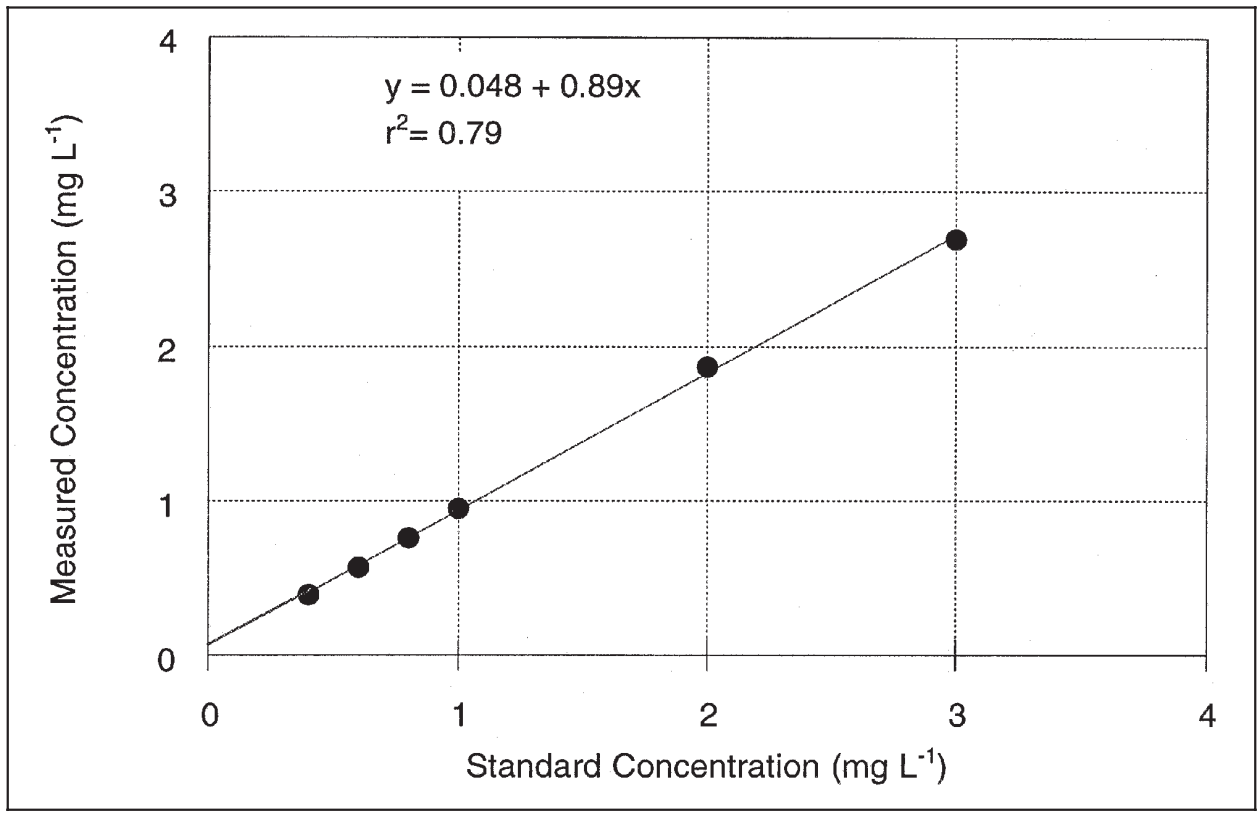

Figure 1. Standard curve for the 1,10 phenanthroline method (Method 1)

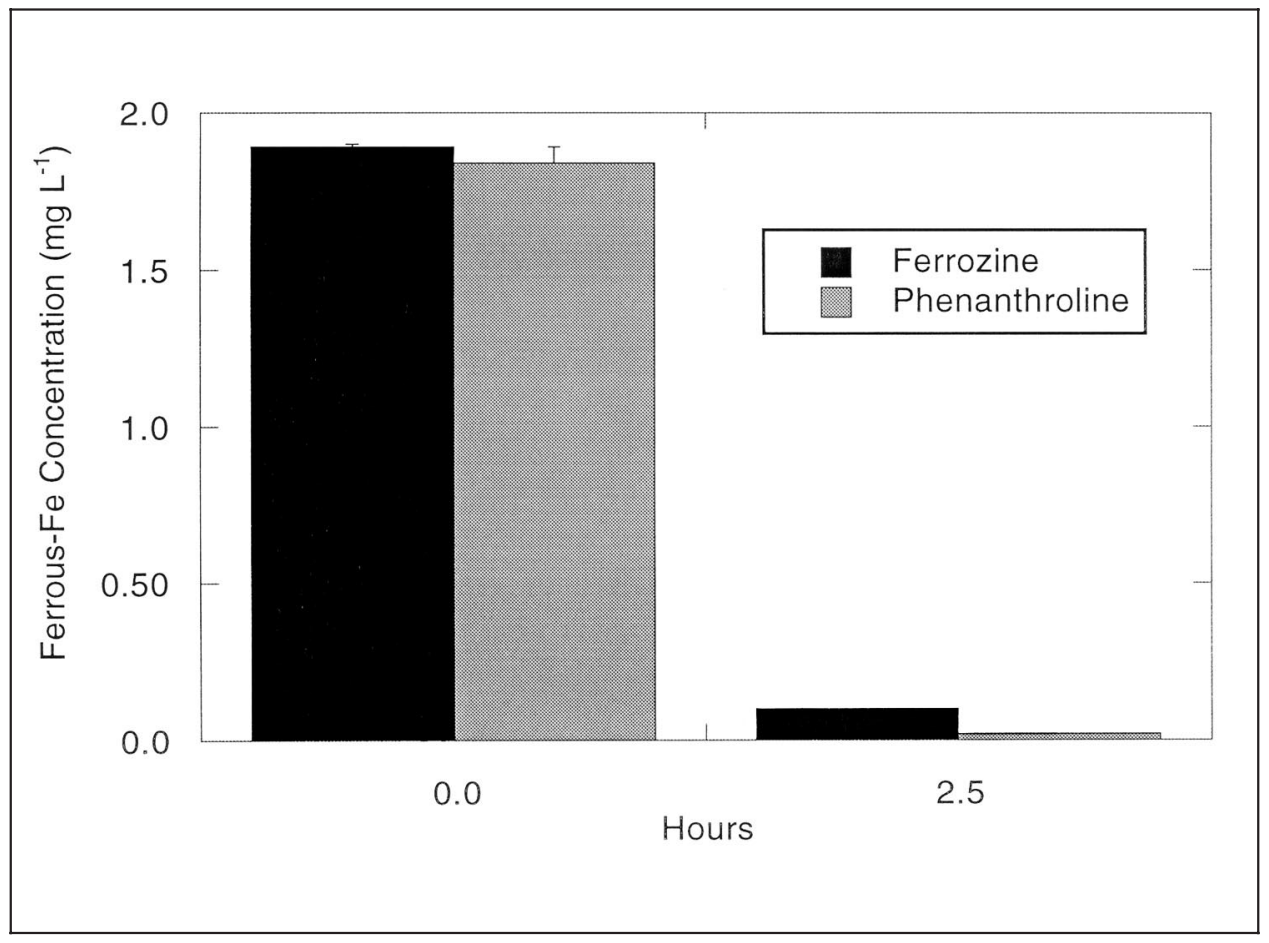

Figure 2. Results of ferrous and ferric iron specificity test 


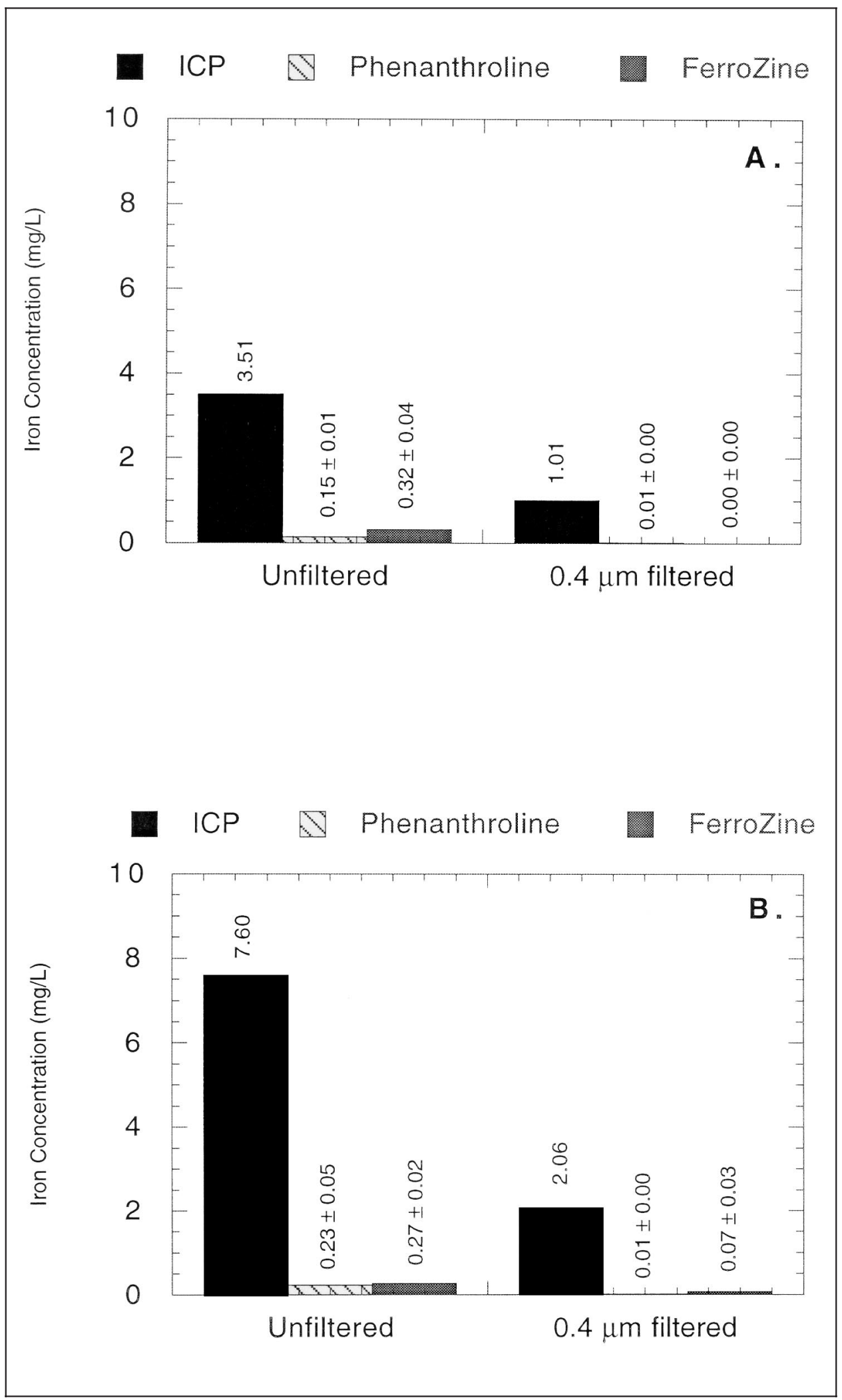

Figure 3. Iron concentrations in oxic Amite River water at low (A) and high (B) concentrations 
Water Quality Technical Note PD-02

March 1999

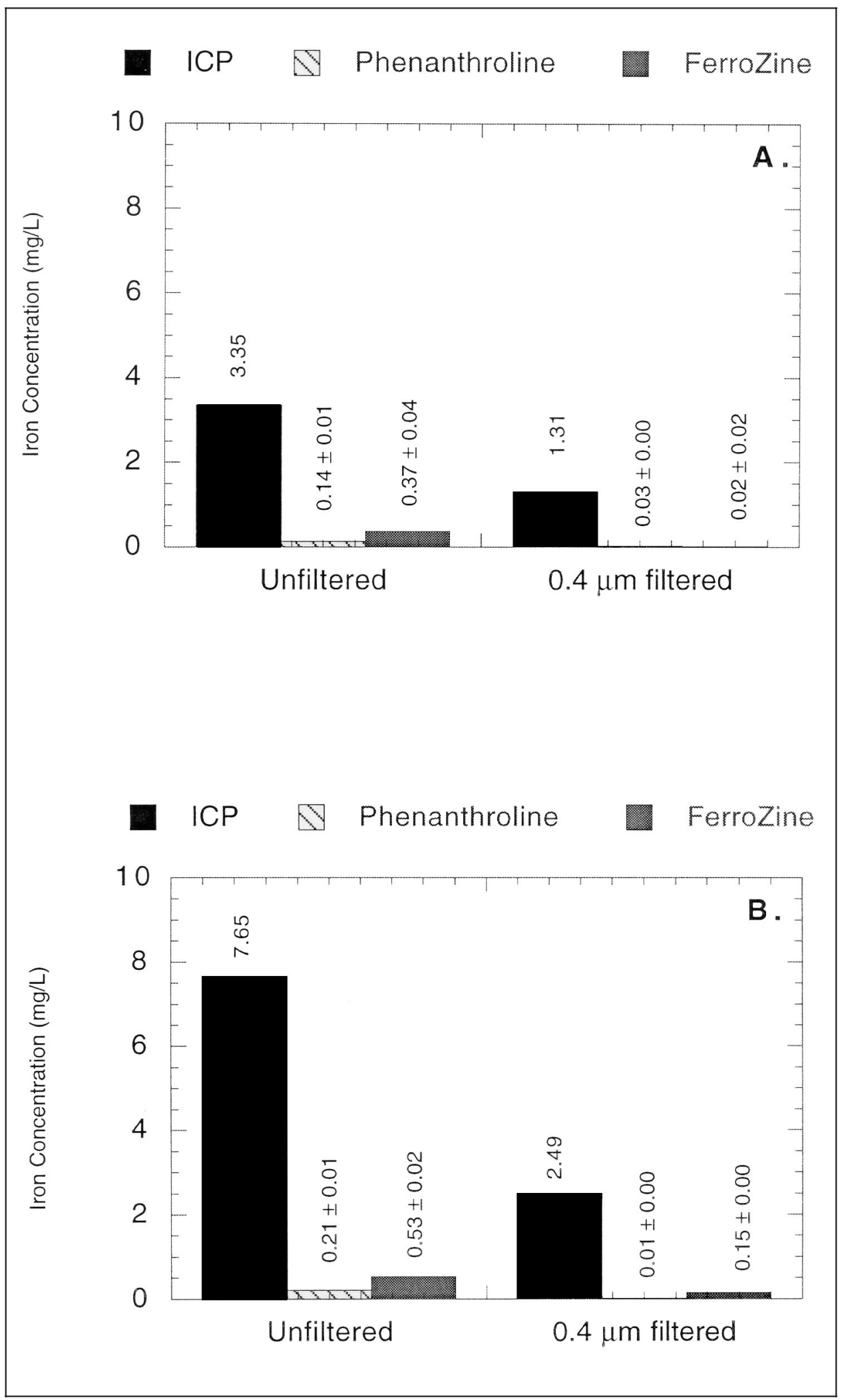

Figure 4. Iron concentrations in partially oxic Amite River water at low (A) and high (B) concentrations 


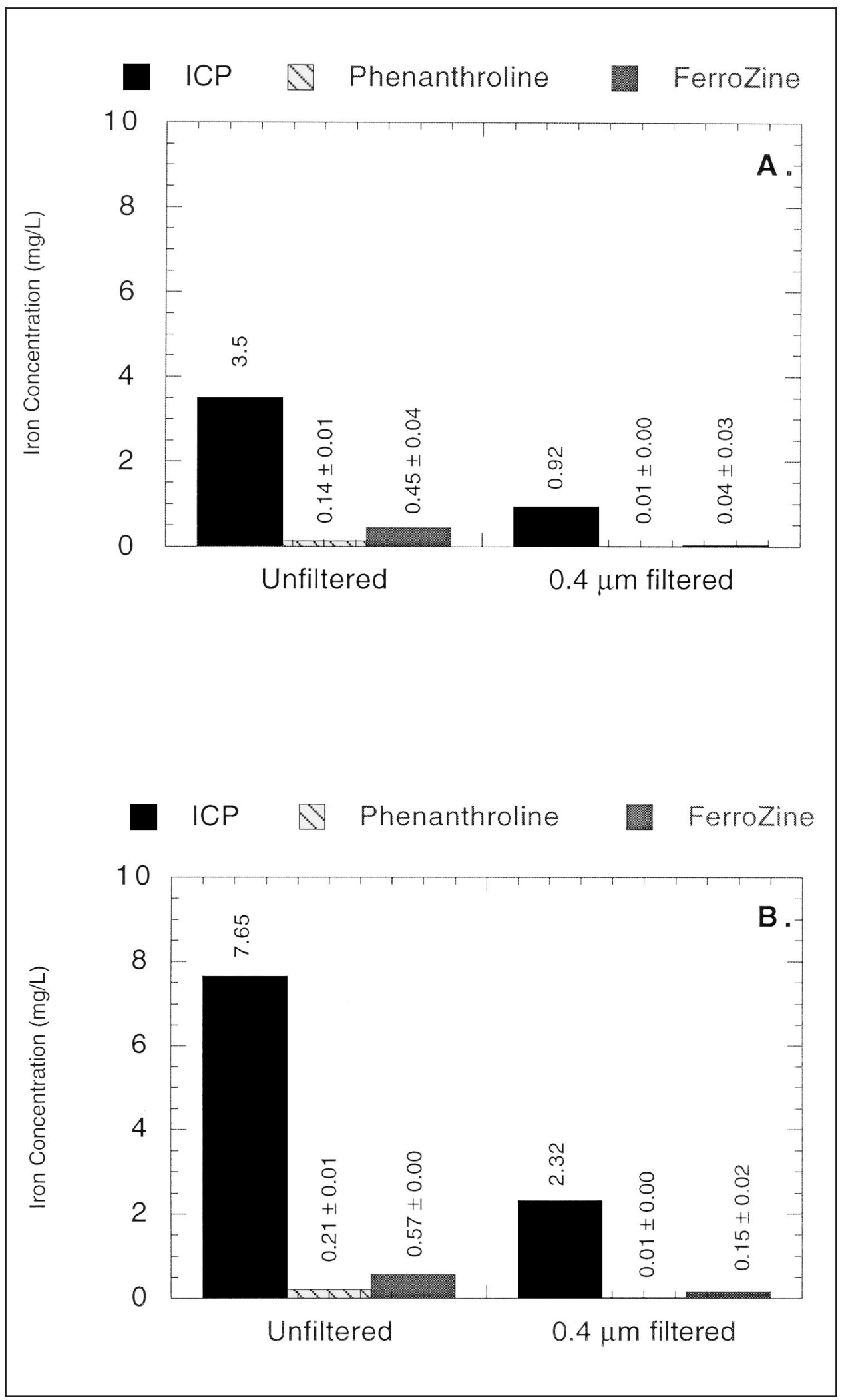

Figure 5. Iron concentrations in anoxic Amite River water at low (A) and high (B) concentrations 
Water Quality Technical Note PD-02

March 1999

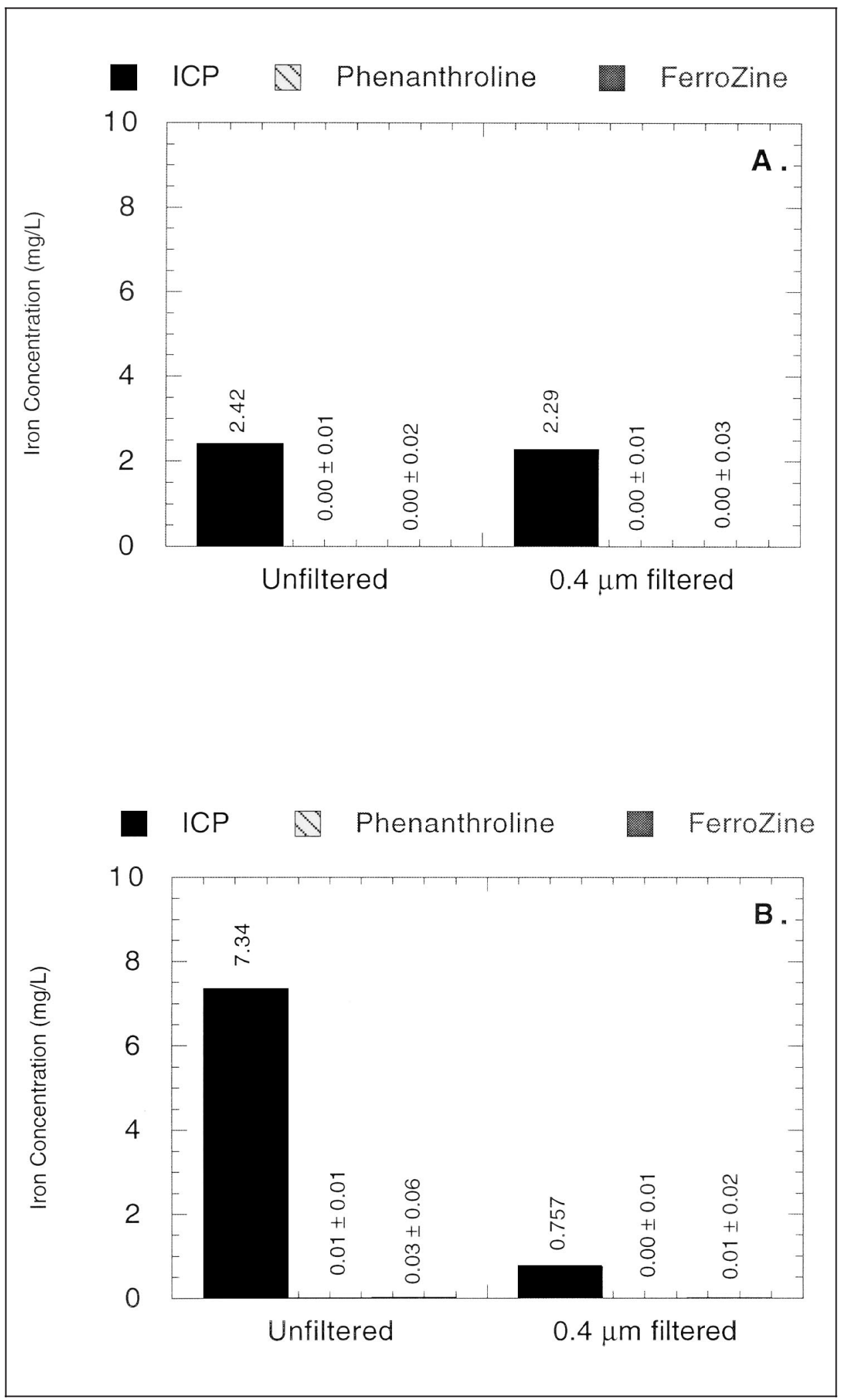

Figure 6. Iron concentrations in oxic simulated reservoir water at low (A) and high (B) concentrations 


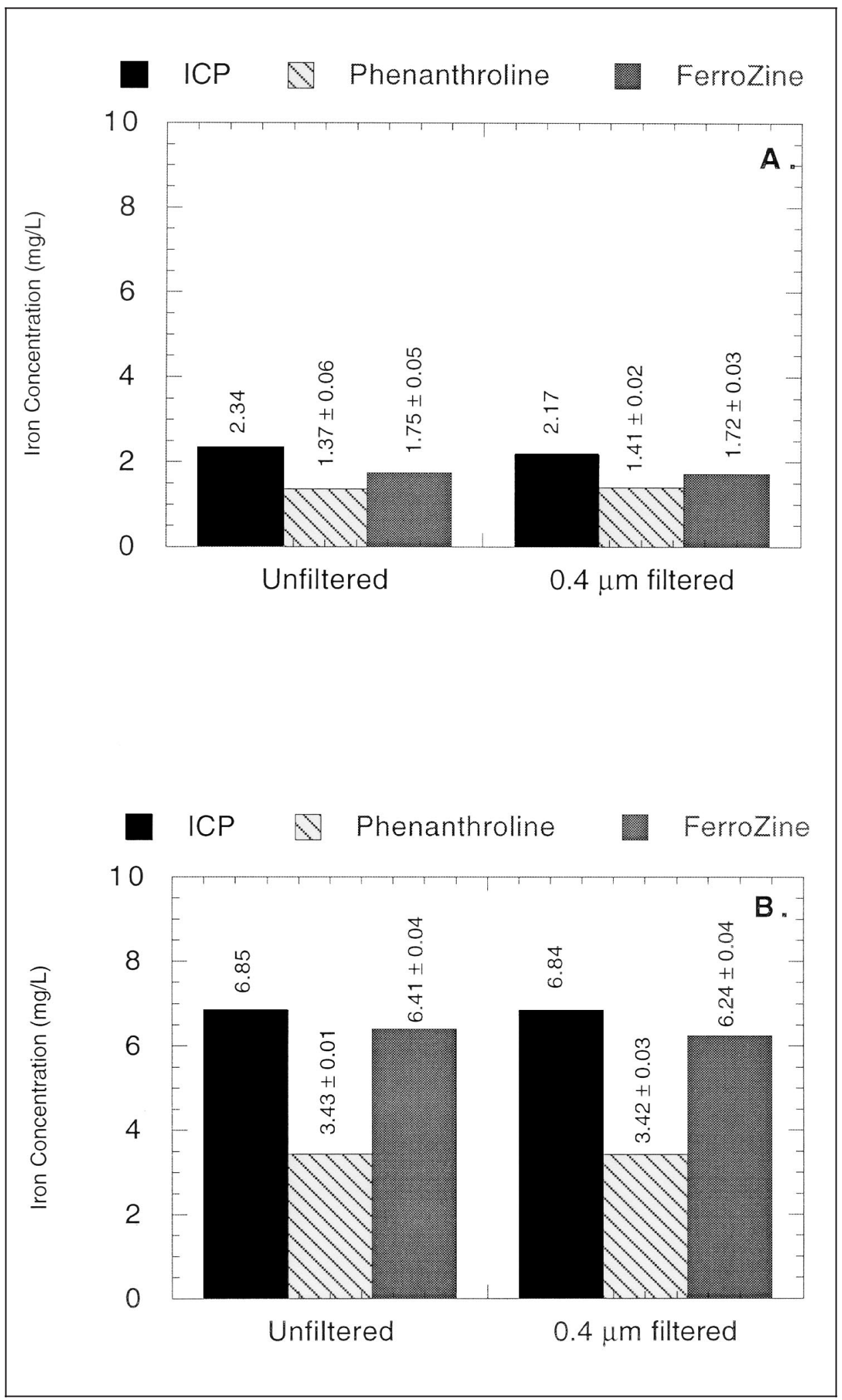

Figure 7. Iron concentrations in partially oxic simulated reservoir water at low $(\mathrm{A})$ and high $(\mathrm{B})$ concentrations 
Water Quality Technical Note PD-02

March 1999

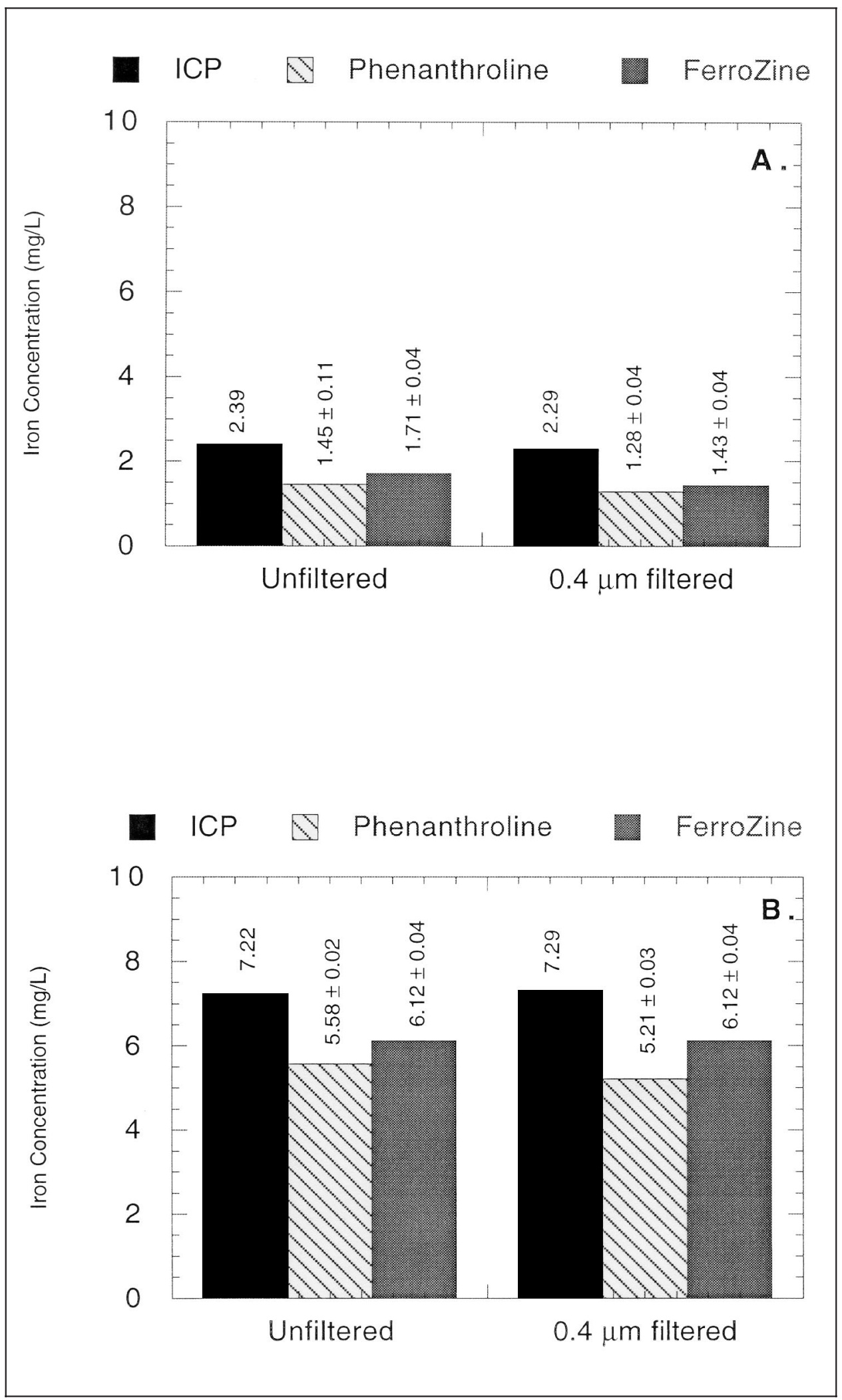

Figure 8. Iron concentrations in anoxic simulated reservoir water at low (A) and high (B) concentrations 
labeled B), source water effects (Amite River, simulated reservoir water), and extent of oxygenation (oxic, partially oxic, and anoxic). Data are presented for ICP analyses (which are considered to represent the total iron available), for Method 1 (using the Hach procedure), and for Method 2 (using FerroZine, "Fz"). Results are shown for both unfiltered samples ("UF") and filtered samples (labeled " 0.4 " for the $0.4-\mu \mathrm{m}$ filters used).

Concentration effects in the amended Amite River samples (Figures 3-5) were not readily discernible, partly due to the low concentrations of ferrous iron in the samples under all conditions of oxygen concentrations. In general, measurements with Method 1 were lower than measurements with Method 2, with differences ranging from near 0 (filtered sample, low range, oxic conditions, Figure 3a) to $0.36 \mathrm{mg} \mathrm{L}^{-1}$ (UF, high range, anoxic conditions, Figure $5 b)$. In all cases, UF samples yielded greater concentrations, suggesting ferrous iron in size fractions greater than $0.4 \mu \mathrm{m}$. In most cases, measurements using Method 1 were approximately 50 percent lower than measurements using Method 2. Effects of oxygen conditions were not discernible between methods or concentrations for the amended Amite River water, although slightly higher concentrations of ferrous iron were observed for the partially oxic and anoxic samples (Figures 4-5).

In the simulated reservoir water samples, ferrous iron was generally below the detection limit (that is, $<0.005 \mathrm{mg} \mathrm{L}^{-1}$ ) under oxic conditions (Figure 6), indicating that oxidation to ferric iron occurred quickly. Thus, comparisons between methods were not possible. In the partially oxic water, concentrations of soluble were lower than concentrations of unfiltered samples but well above the detection limit, indicating ferrous iron (Figure 7). Measurements with Method 1 were lower than measurements using Method 2, especially in the higher concentration sample (Figure 7b). In the anoxic water, responses were similar to those of the partially oxic water, except measurements with Method 1 were much closer to measurements with Method 2 for the higher concentration sample (Figure $8 b$ ). The dissimilar results between methods under partially oxic and anoxic conditions suggest that Method 1 may be a function of oxygen conditions (that is, extent of oxidation of ferrous iron).

Of interest is the detection of ferric iron under oxic conditions in the filtered samples of the simulated reservoir water when compared with measurements in the filtered natural water. The concentrations of anions, cations, and organic compounds, which would be lowest in the low-concentration simulated water, may be a critical factor in the formation of particulate iron compounds that could possibly explain, in part, the observed differences in soluble concentrations. Particle size or choice of filter size may also be a contributing factor. For example, use of a $0.1-\mu \mathrm{m}$ filter may have removed more of the measurable iron, although filter size was not a factor in a similar study (Faulkner, Gambrell, and Ashby 1996). Clearly, the contribution of cations, anions, and organic compounds and concentration effects on iron oxidation and solubility should be considered in assessments of iron oxidation kinetics.

CONCLUSIONS: Both methods (use of 1,10 phenanthroline and FerroZine) were considered to be adequate for specifically determining ferrous iron. In general, FerroZine detected greater concentrations of what was considered to be ferrous iron than did 1,10 phenanthroline. The major difference between the two methods may be attributed to interferences in the 1,10 phenanthroline 
method related to concentrations of cations, anions, and organic compounds and complexation or oxidation under different oxygen conditions.

The method using FerroZine should be considered as the preferred method and is easily conducted in the field. Use of this method (with rapid determination in situ to avoid changes in oxidation states), coupled with measurements of total concentrations on samples that can be preserved for later analysis, will provide adequate measurements of concentrations of ferrous and ferric iron (by difference) in reservoir tailwaters. These data can then be used in modeling applications for predicting downstream concentrations of ferrous and ferric iron.

POINT OF CONTACT: For additional information, contact Mr. Steven L. Ashby at the U.S. Army Engineer Waterways Experiment Station, (601)634-2387, ashbys@mail.wes.army.mil. This technical note should be cited as follows:

Faulkner, S. P., Hintz, P. E., and Ashby, S. L. (1999). "Evaluation of Colorimetric Methods for Measuring Reduced (Ferrous) Iron," Water Quality Technical Notes Collection (WQTN PD-02), U.S. Army Engineer Research and Development Center, Vicksburg, MS. www.wes.army.mil/el/elpubs/wqtncont.html

\section{REFERENCES}

Dortch, M. S., Tillman, D. H., and Bunch, B. W. (1992). "Modeling water quality of reservoir tailwaters," Technical Report W-92-1, U.S. Army Engineer Waterways Experiment Station, Vicksburg, MS.

Faulkner, S. P., Gambrell, R. P., and Ashby, S. L. (1996). "Analytical methods for iron and manganese determinations in reservoir tailwaters: Laboratory investigations," Water Quality Technical Note PD-01, U.S. Army Engineer Waterways Experiment Station, Vicksburg, MS.

Gibbs, C. R. (1976). "Characterization and application of ferrozine iron reagent as a ferrous indicator," Analytical Chemistry 48(8), 1197-1201.

Hach Company. (1992). Water analysis handbook. 2nd ed., Loveland, CO.

Stookey, L. L. (1970). "Ferrozine-a new spectrophotometer reagent for iron,” Analytical Chemistry 42, $779-81$.

Note: The contents of this technical note are not to be used for advertising, publication, or promotional purposes. Citation of trade names does not constitute an official endorsement or approval of the use of such products. 